\title{
Current in open quantum systems
}

\author{
Ralph Gebauer \\ The Abdus Salam International Centre for Theoretical Physics (ICTP), 34014 Trieste, Italy \\ INFM Democritos National Simulation Center, 34013 Trieste, Italy \\ Roberto Car \\ Department of Chemistry and Princeton Materials Institute, \\ Princeton University, Princeton, New Jersey 08540
}

(Dated: September 4, 2018)

\begin{abstract}
We show that a dissipative current component is present in the dynamics generated by a Liouvillemaster equation, in addition to the usual component associated with Hamiltonian evolution. The dissipative component originates from coarse graining in time, implicit in a master equation, and needs to be included to preserve current continuity. We derive an explicit expression for the dissipative current in the context of the Markov approximation. Finally, we illustrate our approach with a simple numerical example, in which a quantum particle is coupled to a harmonic phonon bath and dissipation is described by the Pauli master equation.
\end{abstract}

PACS numbers: 03.65.Yz,72.10.Bg

Open quantum systems are encountered in many domains of physics, ranging from condensed matter physics [1, 2] to quantum optics 3], chemical physics [4] and NMR theory [5]. In all these fields one is interested in the reduced dynamics of a quantum system in contact with its environment. The reduced dynamics is usually approximated by a master equation. A simple example is the Pauli master equation (PME) [6] in which the diagonal elements $\bar{S}_{l l}(t)=\langle l|\bar{S}(t)| l\rangle$ of a coarse grained reduced density operator $\bar{S}(t)$ in the basis of the system eigenstates evolve in time according to:

$$
\frac{d \bar{S}_{l l}}{d t}=\sum_{m \neq l}\left(W_{l m} \bar{S}_{m m}-W_{m l} \bar{S}_{l l}\right) .
$$

The transition probabilities $W_{i j}$ are given by Fermi's golden rule expression in terms of the coupling to the bath. The $W_{i j}$ satisfy detailed balance, which guarantees that the thermal equilibrium distribution is the stationary solution of Eq. (11). A difficulty with Eq. (11) is that it describes a charge (or probability) density evolution that does not seem to satisfy the current continuity equation, given that the current associated with eigenstates is divergenceless. This apparent violation of current continuity makes the use of Eq. (11) problematic to model electron transport kinetics [7, [8], although it has been argued that under steady state conditions Eq. (1) can still be used to simulate electron transport in very small devices [8].

Eq. (1I) is a special case of a Liouville master equation of the form:

$$
\frac{d \bar{S}(t)}{d t}=-i[H, \bar{S}(t)]+\mathcal{C}[\bar{S}(t)],
$$

where $H$ is the Hamiltonian of the system and $\mathcal{C}[\bar{S}(t)]$ describes the dissipative part of the dynamics after eliminating bath memory effects by coarse-graining in time
(Markov approximation) 9, 10. Eq. (2) is derived by assuming a harmonic bath and by treating the system-bath interaction to 2 nd order of perturbation theory. The limits of validity of such approximation and of the Markov approximation requiring separation of the time scales of system and bath, have been amply discussed in the literature [9, 13].

Eq. (2) applies to a generic interacting $N$-particle quantum system described by a $N$-particle coarsegrained reduced density operator $\bar{S}$.

In what follows we adopt atomic units $(e=m=$ $\hbar=1)$. We indicate coarse-grained quantities by a bar. The coarse-grained charge density $\bar{n}(\boldsymbol{x} ; t)$ is given by $\bar{n}(\boldsymbol{x} ; t)=\operatorname{Tr}\{\bar{S}(t) \hat{\rho}(\boldsymbol{x})\}$ in terms of the charge density operator $\hat{\rho}(\boldsymbol{x})=\sum_{l=1, N} \delta\left(\boldsymbol{x}-\hat{\boldsymbol{x}}_{l}\right)$. The coarse-grained Hamiltonian current density is given by $\overline{\boldsymbol{\jmath}}_{H}(\boldsymbol{x} ; t)=$ $\operatorname{Tr}\{\bar{S}(t) \hat{\boldsymbol{J}}(\boldsymbol{x})\}$, in terms of the current density operator $\hat{\boldsymbol{J}}(\boldsymbol{x})=\frac{1}{2} \sum_{l=1, N}\left[\hat{\boldsymbol{p}}_{l} \delta\left(\boldsymbol{x}-\hat{\boldsymbol{x}}_{l}\right)+\delta\left(\boldsymbol{x}-\hat{\boldsymbol{x}}_{l}\right) \hat{\boldsymbol{p}}_{l}\right]$. If the potential in $H$ is local in space, then the continuity equation $\dot{\bar{n}}(\boldsymbol{x} ; t)=-\nabla \cdot \overline{\boldsymbol{\jmath}}_{H}(\boldsymbol{x} ; t)$ is evidently satisfied if [7]:

$$
\operatorname{Tr}\{\mathcal{C}[\bar{S}(t)] \hat{\rho}(\boldsymbol{x})\}=0 .
$$

Generally master equations in which the dissipative dynamics leads asymptotically to the thermal equilibrium distribution do not satisfy Eq. (3) [18]. This is at variance with the exact reduced dynamics which satisfies the continuity equation if the coupling to the bath is local.

In this letter we show that assertions on the violation of continuity by master equations that do not satisfy Eq. (3) are indeed incorrect, and that the coarse-grained (Markovian) dynamics exactly satisfies current continuity, as the true reduced dynamics does if the coupling to the bath is local. This is because the following equation holds:

$$
\operatorname{Tr}\{\mathcal{C}[\bar{S}(t)] \hat{\rho}(\boldsymbol{x})\}=\dot{\bar{n}}_{\mathcal{C}}(\boldsymbol{x})=-\nabla \cdot \overline{\boldsymbol{\jmath}}_{\mathcal{C}}(\boldsymbol{x} ; t)
$$


In Eq. (4) $\bar{\jmath}_{\mathcal{C}}$ is a dissipative current originating from the action of the bath on the system on the coarse grained time scale of Markovian dynamics. This current contribution, which has been ignored so far, is of the same order of the Hamiltonian current in strongly inhomogeneous systems. We also provide an expression for $\overline{\boldsymbol{\jmath}}_{\mathcal{C}}$, whose calculation only requires knowledge of the coarse-grained density operator $\bar{S}(t)$, of the eigenvalues and eigenvectors of the system Hamiltonian $H$, of the Hamiltonian $R$ of the harmonic reservoir, and of the coupling $K$ between the system and the bath.

The derivation of a master equation of the form (2) can be found in textbooks [10]. Here we limit ourselves to pointing out the key elements of this derivation that are important for our discussion of current continuity.

Let $\mathrm{S}(\mathrm{t})$ be the reduced density operator in absence of coarse graining. As usual, to separate the effect of the bath from system dynamics, it is convenient to work in the interaction picture (IP): $S(t)=$ $e^{-i H\left(t-t_{0}\right)} s(t) e^{i H\left(t-t_{0}\right)}$, where $t_{0}$ is a time preceding $t$ and $s(t)$ is the reduced density operator in the IP. Taking the time derivative of $S(t)$, the reduced density operator in the Schrödinger picture (SP), one gets:

$$
\frac{d S(t)}{d t}=-i[H, S(t)]+e^{-i H\left(t-t_{0}\right)} \frac{d s(t)}{d t} e^{i H\left(t-t_{0}\right)} .
$$

Eq. (5) governs the exact dynamics of the system. The first term on the right hand side (r.h.s.) corresponds to the usual Hamiltonian dynamics, which satisfies current continuity if the potentials in $H$ are local. For the second term on the r.h.s. one can show that $\operatorname{Tr}\left\{e^{-i H\left(t-t_{0}\right)} \frac{d s(t)}{d t} e^{i H\left(t-t_{0}\right)} \hat{\rho}(\boldsymbol{x})\right\}=0$, if the interaction potential with the bath is local. Therefore, this term does not modify the instantaneous distribution of charges, and the exact dynamics satisfies current continuity.

In order to convert Eq. (5) into a master equation of the form (2), one replaces the time-derivative $\frac{d s(t)}{d t}$ in (5D) by a finite difference approximation $\frac{\Delta s}{\Delta t}=\frac{s(t)-s\left(t_{0}\right)}{t-t_{0}}$ calculated to leading order of perturbation theory using Fermi's golden rule, and then one takes the limit $\Delta t \rightarrow 0$ (corresponding to coarse-graining in time) in the equation for system dynamics, i.e. one writes for the coarsegrained density operator $\bar{S}(t)$ :

$$
\begin{aligned}
\frac{d \bar{S}(t)}{d t} & =-i[H, \bar{S}(t)]+\lim _{t_{0} \rightarrow t} e^{-i H\left(t-t_{0}\right)} \frac{\Delta s}{\Delta t} e^{i H\left(t-t_{0}\right)} \\
& =-i[H, \bar{S}(t)]+\lim _{t_{0} \rightarrow t} \frac{\Delta s}{\Delta t}
\end{aligned}
$$

We recall that Fermi's golden rule expression for $\frac{\Delta s}{\Delta t}$ is independent of $\Delta t$. The limit for $\Delta t \rightarrow 0$ is possible because $\Delta t$, although large relative to inverse system frequencies for Fermi's golden rule to be applicable, is small compared to the relaxation time. Thereafter all reference to $t_{0}$ disappears from Eq. (6) and the future evolution of the system is entirely determined by its present and not by its past (Markov approximation). Eq. (6) is the same as Eq. (2) 9, 10.

To prove that Eq. (2) satisfies charge continuity even when Eq. (3) is not satisfied, we compute the time derivative of the coarse-grained charge density:

$$
\begin{aligned}
\frac{d \bar{n}(\boldsymbol{x})}{d t} & =\operatorname{Tr}\left\{\frac{d \bar{S}(t)}{d t} \hat{\rho}(\boldsymbol{x})\right\} \\
& =-i \operatorname{Tr}\{[H, \bar{S}(t)] \hat{\rho}(\boldsymbol{x})\}+\operatorname{Tr}\left\{\lim _{\Delta t \rightarrow 0} \frac{\Delta s}{\Delta t} \hat{\rho}(\boldsymbol{x})\right\} .
\end{aligned}
$$

The first term on the r.h.s. of Eq. (7) gives: $-i \operatorname{Tr}\{[H, \bar{S}(t)] \hat{\rho}(\boldsymbol{x})\}=-\nabla \cdot \overline{\boldsymbol{\jmath}}_{H}(x ; t)$. To compute the remaining term we consider the exact density operator $S\left(t^{\prime}\right)=e^{-i H\left(t^{\prime}-t_{0}\right)} s\left(t^{\prime}\right) e^{i H\left(t^{\prime}-t_{0}\right)}$, whose dynamics includes the effect of the bath, and the density operator $S_{H}\left(t^{\prime}\right)$, whose dynamics is governed by $H$ alone and does not include the effect of the bath: $S_{H}\left(t^{\prime}\right)=$ $e^{-i H\left(t^{\prime}-t_{0}\right)} s(t) e^{i H\left(t^{\prime}-t_{0}\right)}$. Here we have assumed that at time $t S(t)=S_{H}(t)$. Then at time $t_{0}, S\left(t_{0}\right)=s\left(t_{0}\right)$ and $S_{H}\left(t_{0}\right)=s(t)$. Thus:

$$
\begin{aligned}
\Delta s & =s(t)-s\left(t_{0}\right)=S(t)-S\left(t_{0}\right)-S_{H}(t)+S_{H}\left(t_{0}\right) \\
& =\int_{t_{0}}^{t} d t^{\prime}\left(\dot{S}\left(t^{\prime}\right)-\dot{S}_{H}\left(t^{\prime}\right)\right)
\end{aligned}
$$

Since both the exact and the $H$-dynamics satisfy continuity, we can write

$$
\begin{aligned}
\operatorname{Tr}\{\Delta s \hat{\rho}(\boldsymbol{x})\} & =\int_{t_{0}}^{t} d t^{\prime}\left(\dot{n}\left(\boldsymbol{x} ; t^{\prime}\right)-\dot{n}_{H}\left(\boldsymbol{x} ; t^{\prime}\right)\right) \\
& =-\nabla \cdot \int_{t_{0}}^{t} d t^{\prime}\left(\boldsymbol{j}\left(\boldsymbol{x} ; t^{\prime}\right)-\boldsymbol{j}_{H}\left(\boldsymbol{x} ; t^{\prime}\right)\right) \\
& =-\nabla \cdot \int_{t_{0}}^{t} d t^{\prime} \boldsymbol{j}_{\mathcal{C}}\left(\boldsymbol{x} ; t^{\prime}\right),
\end{aligned}
$$

where $\boldsymbol{j}_{H}=\operatorname{Tr}\left\{S_{H} \hat{\boldsymbol{J}}\right\}$ and $\boldsymbol{j}=\operatorname{Tr}\{S \hat{\boldsymbol{J}}\}$. In Eq. (9) the difference between the exact current, which includes the effect of the bath, and the $H$-current defines the current $\boldsymbol{j}_{\mathcal{C}} \equiv \boldsymbol{j}-\boldsymbol{j}_{H}$. Using (9) we obtain the second term on the r.h.s. of Eq. (7):

$$
\begin{aligned}
\lim _{\Delta t \rightarrow 0} & \operatorname{Tr}\left\{\frac{\Delta s}{\Delta t} \hat{\rho}(\boldsymbol{x})\right\}=-\lim _{t_{0} \rightarrow t} \frac{1}{\Delta t} \nabla \cdot \int_{t_{0}}^{t} d t^{\prime} \boldsymbol{j}_{\mathcal{C}}(\boldsymbol{x} ; t) \\
= & -\nabla \cdot\left(\lim _{t_{0} \rightarrow t} \frac{1}{\Delta t} \int_{t_{0}}^{t} d t^{\prime} \boldsymbol{j}_{\mathcal{C}}(\boldsymbol{x} ; t)\right)
\end{aligned}
$$

We identify the term in parenthesis in Eq. (10) with the coarse-grained current $\overline{\boldsymbol{\jmath}}_{\mathcal{C}}$. Thus we obtain

$$
\frac{d \bar{n}(\boldsymbol{x})}{d t}=-\nabla \cdot \overline{\boldsymbol{\jmath}}_{H}(\boldsymbol{x} ; t)-\nabla \cdot \overline{\boldsymbol{\jmath}}_{\mathcal{C}}(\boldsymbol{x} ; t)=-\nabla \cdot \overline{\boldsymbol{\jmath}}(x ; t) .
$$

Eq. (11) is the main result of this Letter: it shows that the master equation does not violate current continuity and that the coarse-grained physical current $\overline{\boldsymbol{\jmath}}$, i.e. 
the current measured in experiments, is the sum of two contributions, a current $\overline{\boldsymbol{\jmath}}_{H}$, associated with the charge flow from Hamiltonian propagation in the Liouville master equation (21), and a current $\overline{\boldsymbol{\jmath}}_{\mathcal{C}}$, associated with the charge flow due to inelastic collisions with the bath in the same equation. In the following we show that the limit in Eq. (10) can be calculated to leading order of perturbation theory, i.e. at the same level of approximation used in the derivation of the master equation itself. The resulting expression for $\overline{\mathcal{J}}_{\mathcal{C}}$ is independent of $t_{0}$. Thus $\overline{\boldsymbol{\jmath}}(x ; t)$ can be calculated from the knowledge of $\bar{S}(t)$ at time $t$ only.

For the derivation of an explicit expression for $\overline{\mathcal{J}}_{\mathcal{C}}$, we consider a coupling $K$ of the form $K=\sum_{\alpha} V^{\alpha} F^{\alpha}$, where $V^{\alpha}=\sum_{l=1, N} V_{l}^{\alpha}\left(\hat{\boldsymbol{x}}_{l}\right)$ is a local potential acting on the system, and $F^{\alpha}$ acts on bath variables only. The sum is over all the bath eigenmodes (phonons), but to simplify the notation we drop the index $\alpha$ in what follows. A standard procedure 10 gives $\Delta s$ to second order of perturbation theory:

$$
\begin{aligned}
\Delta s=s(t)-s\left(t_{0}\right)= & -\sum_{m n o p}\left\{A_{m n, o p} w_{o p}^{-}-B_{m n, o p} w_{m n}^{+}\right\} \\
& \times \int_{0}^{t-t_{0}} \mathrm{~d} \xi e^{i\left(e_{m}-e_{n}+e_{o}-e_{p}\right) \xi}, \quad(12)
\end{aligned}
$$

where the sums are over eigenstates $|i\rangle$ of $H$ having energy $e_{i}$. The operators $A$ and $B$ are:

$$
\begin{aligned}
A_{m n, o p}= & |m\rangle V_{m n}\langle n \mid o\rangle V_{o p}\langle p| s\left(t_{0}\right) \\
& -|o\rangle V_{o p}\left\langle p\left|s\left(t_{0}\right)\right| m\right\rangle V_{m n}\langle n|, \\
B_{m n, o p}= & |o\rangle V_{o p}\left\langle p\left|s\left(t_{0}\right)\right| m\right\rangle V_{m n}\langle n| \\
& -s\left(t_{0}\right)|m\rangle V_{m n}\langle n \mid o\rangle V_{o p}\langle p|,
\end{aligned}
$$

where $V_{m n}=\langle m|V| n\rangle$, and

$$
\begin{aligned}
w_{m n}^{+} & =\int_{0}^{\infty} e^{i\left(e_{m}-e_{n}\right) t^{\prime}}\left\langle F\left(t^{\prime}\right) F(0)\right\rangle_{R} \mathrm{~d} t^{\prime}, \\
w_{o p}^{-} & =\int_{0}^{\infty} e^{i\left(e_{o}-e_{p}\right) t^{\prime}}\left\langle F(0) F\left(t^{\prime}\right)\right\rangle_{R} \mathrm{~d} t^{\prime}
\end{aligned}
$$

are bath spectral densities, which, for a harmonic bath, can be calculated analytically and are zero when $m=n$ $(o=p)$. In Eq. (14) $F\left(t^{\prime}\right)$ is in the IP and $\langle\cdots\rangle_{R}$ denotes an average over the bath degrees of freedom. If $t-t_{0}$ is large compared to the inverse level spacing $1 /\left(e_{i}-e_{j}\right)$, the time integral in Eq. (12) leads to selection rules, where the only terms that survive are those with $m=p$ and $n=$ $o$. The resulting $\Delta s$ varies linearly with $\Delta t$. Inserting $\frac{\Delta s}{\Delta t}$ in Eq. (6), one obtains the usual expression for the dissipative master equation [10].

In order to compute the dissipative current, one has to evaluate

$$
\bar{\jmath}_{\mathcal{C}}(\boldsymbol{x}, t)=\lim _{t_{0} \rightarrow t} \frac{1}{\Delta t} \int_{t_{0}}^{t} d t^{\prime} \operatorname{Tr}\left\{\left(S\left(t^{\prime}\right)-S_{H}\left(t^{\prime}\right)\right) \boldsymbol{J}(\boldsymbol{x})\right\} .
$$

Using $\quad\left(S\left(t^{\prime}\right)-S_{H}\left(t^{\prime}\right)\right)=$ $e^{-i H\left(t^{\prime}-t_{0}\right)}\left(s\left(t^{\prime}\right)-s\left(t_{0}\right)-\left(s(t)-s\left(t_{0}\right)\right)\right) e^{i H\left(t^{\prime}-t_{0}\right)}$, one can insert the expression (12) for $s\left(t^{\prime}\right)-s\left(t_{0}\right)$ and $s(t)-s\left(t_{0}\right)$, respectively, into Eq. (15).

The additional time-integral and the exponentials lead to different selection rules than in case of Eq. (12). Identifying the leading terms, and finally letting $t_{0} \rightarrow t$, we obtain an expression for the dissipative current:

$$
\overline{\boldsymbol{J}}_{\mathcal{C}}(\boldsymbol{x} ; t)=i \sum_{k \neq l} \frac{1}{e_{l}-e_{k}}\left(\mathcal{T}_{k l}-\mathcal{R}_{k l}\right) \hat{\boldsymbol{J}}(\boldsymbol{x})_{l k}
$$

where $\hat{\boldsymbol{J}}(\boldsymbol{x})_{l k} \equiv\langle l|\hat{\boldsymbol{J}}(\boldsymbol{x})| k\rangle$ and $\mathcal{R}_{k l}, \mathcal{T}_{k l}$ are given by:

$$
\begin{aligned}
\mathcal{R}_{k l}= & -\sum_{n \neq k}\left|V_{n k}\right|^{2} \bar{S}_{k l}(t) w_{n k}^{-} \\
& -\sum_{n \neq l} \bar{S}_{k l}(t)\left|V_{l n}\right|^{2} w_{l n}^{+}, \\
\mathcal{T}_{k l}= & -\sum_{n \neq k} V_{k n} V_{n l} \bar{S}_{l l}(t) w_{n l}^{-} \\
& +\sum_{n \neq k} V_{k n} \bar{S}_{n n} V_{n l}\left(w_{n l}^{+}+w_{k n}^{-}\right) \\
& -\sum_{n \neq l} \bar{S}_{k k}(t) V_{k n} V_{n l} w_{k n}^{+},
\end{aligned}
$$

Eq. (16) is the second important result of this paper and completes the proof that $\overline{\boldsymbol{\jmath}}_{\mathcal{C}}(\boldsymbol{x} ; t)$ can be calculated from knowledge of $\bar{S}(t)$ and microscopic Hamiltonian only. Eq. (16) gives (to 2nd order of perturbation theory) the coarse-grained current associated to phonon induced inelastic transitions between all pairs of system states. The transition matrix elements depend on (amplitude and phase of) the coupling potential and on the fluctuations of the bath (via the bath spectral densities). The derivation of Eq. (16) does not require additional assumptions besides those made in the derivation of the Liouville master equation. In this context Eq. (16) is exact. Our approach requires an explicit phonon (bath) model, however simplified.

Sometimes master equations are used without reference to an explicit bath model. This is the case, for instance, with the common relaxation time approximation. This approximation does not violate charge continuity when it is applied to spatially homogeneous systems. In inhomogeneous systems, however, the relaxation time approximation leads to violation of charge continuity whenever the bath originates a spatially non-uniform instantaneous change of the coarse-grained charge density, i.e. whenever $\dot{\bar{n}}_{\mathcal{C}}(\boldsymbol{x}) \neq 0$ [11].

Finally, we use a simple numerical example to illustrate our theory. In this example a single quantum particle (electron) is in a one dimensional potential $U(x)$. The electron is initially placed in the first excited eigenstate 

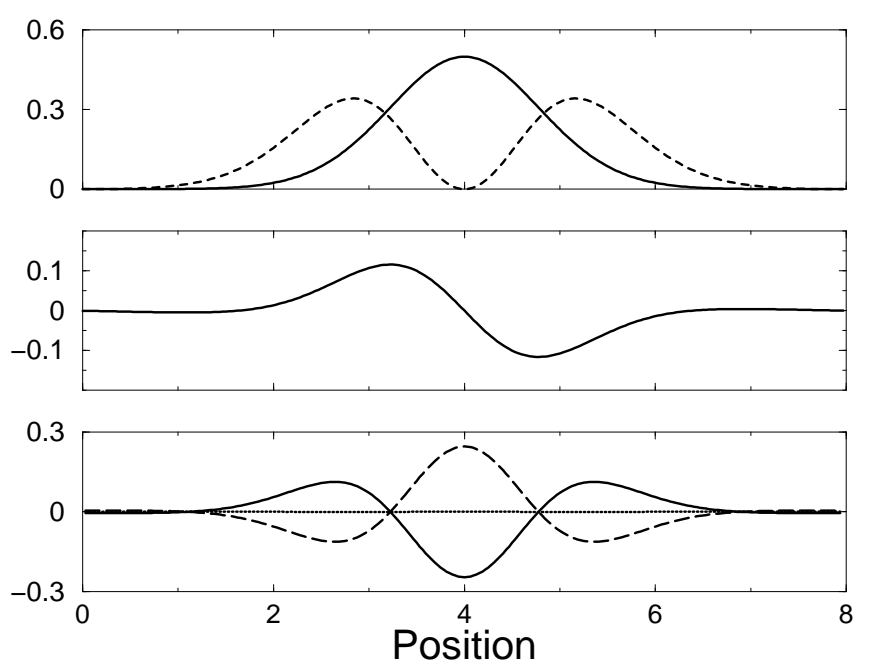

FIG. 1: Upper Panel: Calculated charge densities of the ground state (solid line) and first excited state (dashed line). Middle Panel: Dissipative current $\bar{\jmath}_{\mathcal{C}}(x)$ at a given instant of the propagation, calculated from Eq. (16). Lower panel: Divergence of $\bar{\jmath}_{\mathcal{C}}(x)$ (solid line), time derivative of the charge density $\dot{\bar{n}}(x ; t)$ at the same time (dashed line), and the sum of the two quantities (dotted line). It is evident that continuity equation is satisfied during relaxation.

of the Hamiltonian $H=-\frac{1}{2} \frac{d^{2}}{d x^{2}}+U(x)$. In absence of dissipation the electron would stay in the excited state, but due to coupling with a phonon bath it relaxes toward thermal equilibrium. In this case, neglecting the imaginary part of the bath spectral densities, Eq. (2) reduces to Eq. (11), i.e. the PME, and the density matrix stays diagonal while the system approaches equilibrium. Even though the density matrix does not carry any current, Eq. (16) gives a non-zero current due to the $\mathcal{T}_{k l}$ terms during relaxation dynamics.

We take

$$
w_{n m}^{+}=w_{m n}^{-}=\left\{\begin{array}{ll}
\bar{n}\left(e_{n}-e_{m}\right)+1 & e_{n}>e_{m} \\
\bar{n}\left(e_{m}-e_{n}\right) & e_{n}<e_{m}
\end{array},\right.
$$

where $\bar{n}(\omega)=1 /\left(e^{\frac{\omega}{k T}}-1\right)$ is the mean occupation number of phonons with energy $\omega$. This simple choice guarantees detailed balance. We assume that $V \equiv \frac{d U(x)}{d x}$ is the potential that characterizes the coupling with the bath. The transition probabilities in Eq. (1) are given by $W_{l m}=2\left|V_{l m}\right|^{2} w_{m l}^{+}$. We represent the space coordinate $x$ on a fine numerical grid with 256 points. In Fig. 1 we report the calculated charge density $n_{0}(x)$ of the ground state, the calculated charge density $n_{1}(x)$ of the first excited state, and the calculated time derivative of the charge density $\dot{\bar{n}}(x ; t)$ at a time $t$ during relaxation dynamics (in this example we take $k T=0.4\left(e_{1}-e_{0}\right)$ ). It is clear that there can be no current associated to the eigenstates to compensate the change of charge density $\dot{\bar{n}}(x ; t)$.

In the same figure, we report the dissipative current density $\bar{\jmath}_{\mathcal{C}}(x ; t)$ at the same time $t$. It is obtained from Eq. (16), where $\mathcal{R}_{k l}=0$ because $\bar{S}(t)$ is diagonal. As we can see, the continuity equation $\dot{\bar{n}}(x ; t)+\frac{d}{d x} \bar{\jmath}_{\mathcal{C}}(x ; t)=0$ is exactly satisfied within the numerical accuracy of the calculation.

Numerical integration of Eq. (2) for a single quantum particle is feasible, but is not possible in general for interacting many-body quantum systems. In practical applications to a many-body quantum system, the latter is usually approximated by a system of non-interacting particles in an effective single-particle Hamiltonian like e.g. the time dependent Hartree Hamiltonian [15]. Then Eq. (2), the $N$-particle Liouville master equation, can be reduced to an equation for an effective single-particle density operator in which the master term depends quadratically rather than linearly on the (single-particle) density operator 16. The basic assumptions on perturbative coupling to the bath and coarse-graining in time that led to Eq. (21) remain valid, however, and so is our analysis that led to Eq. (16). Also in this case, a proper expression for $\overline{\mathcal{J}}_{\mathcal{C}}(\boldsymbol{x} ; t)$ can be found, which restores exactly current continuity [1], removing a major limitation to the application of quantum kinetic approaches to the study of electron transport problems.

We thank Morrel Cohen, Erio Tosatti, Kieron Burke, and David Vanderbilt for many stimulating discussions. This work has been partially supported by DOE under grant DE-FG02-01ER45928 and by ONR under grant N00014-01-1-1061.

[1] R.P. Feynman and F.L. Vernon, Ann. Phys. 24, 118 (1963).

[2] A.O. Caldeira and A.J. Leggett, Physica A 121, 587 (1983).

[3] H. Haken, Laser Theory (Springer, Berlin, 1970).

[4] N.G. van Kampen, Stochastic Processes in Physics and Chemistry (North-Holland, Amsterdam, 1992).

[5] A.G. Redfield, IBM J. Res. Dev. 1, 19 (1957).

[6] W. Pauli, Festschrift zum 60. Geburtstage A. Sommerfeld (Hirzel, Leipzig, 1928), p. 30.

[7] W.R. Frensley, Rev. Mod. Phys. 62, 745 (1990).

[8] M.V. Fischetti, J. Appl. Phys. 83, 270 (1998).

[9] C. Cohen-Tannoudji, J. Dupont-Roc, and G. Grynberg, Atom-photon interactions: basic processes and applications, Wiley, New York (1992).

[10] W.H. Louisell, Quantum Statistical Properties of Radiation (Wiley, New York, 1973).

[11] H. Mizuta and G.J. Goodings, J. Phys.: Cond. Mat. 3, 3739 (1991).

[12] R. Gebauer and R. Car, submitted.

[13] R.K. Wangness and F. Bloch, Phys. Rev. 89, 728 (1953).

[14] M.V. Fischetti, Phys. Rev. B 59, 4901 (1999).

[15] P.A.M. Dirac, Proc. Cambr. Philos. Soc. 26, 376 (1930), A.D. McLachlan, Mol. Phys. 8, 39 (1964).

[16] R. Hübner, R. Graham, Phys. Rev. B 53, 4870 (1996).

[17] R. Gebauer and R. Car, in preparation. 
[18] A remarkable exception being the Boltzmann transport equation, where the scattering occurs between

planewaves. 